\title{
A study on estimation of serum ferritin in type 2 diabetes mellitus cases on oral hypoglycemic agents
}

\author{
Bhaktavachalam Prudhvi ${ }^{1}$, Boddepalli Venkataramarao ${ }^{2, *}$ \\ ${ }^{\mathbf{1}}$ Assistant Professor, ${ }^{2}$ Professor, Dept. of Biochemistry, ACSR Medical College, Nellore, Andhra Pradesh, India \\ *Corresponding Author: \\ Email: sujatha2481@gmail.com
}

\begin{abstract}
Introduction: Diabetes mellitus is a metabolic disorder characterized by hyperglycemia and associated micro and macro vascular complications on long duration. Insulin resistance is associated with onset of type 2 DM and various studies point its connection to iron overload in the body. Hyperinsulinemia due to resistance to insulin may be responsible for increasing serum Ferritin.

Objectives: The present study was carried out to determine the relationship between serum Ferritin and type 2 diabetes mellitus and to establish correlation between serum Ferritin and fasting blood glucose as well as with HbA1c.

Materials and Methods: An observational study for one year at a tertiary care hospital was carried on 50 diabetic male cases and 50 age matched controls. BMI, serum ferritin, HbA1c, FBS and PPBS were estimated on all the cases and controls as per standard guidelines and compared between cases and controls. P value less than 0.05 was considered statistically significant.

Results: In the present study, 41-50 years was the common age group, and BMI $\left(\mathrm{kg} / \mathrm{m}^{2}\right)$, Fasting blood sugar, Post prandial blood sugar parameters were statistically significant between cases and controls. The mean HbA1c\% in cases was $9.48 \pm 2.65$ and greater than controls and significant correlation with serum ferritin levels which was $359.11 \pm 87.18$ was observed. Greater levels of Serum ferritin was observed in cases with elevated levels of $\mathrm{HbA} 1 \mathrm{c} \%$. A strong positive correlation was found between serum ferritin and HbA1c, FBS and PPBS levels in the study.

Discussion: This study clearly explains that there is a significant increase in the serum ferritin in cases of diabetes mellitus compared to normal controls and hyperferritenemia may be one of the causes for decreased insulin production and development of insulin resistance in diabetic individuals. Study of iron and related parameters may be a useful offshoot of the conventional studies on diabetes and its complications. Hence monitoring the prevalence of serum ferritin is beneficial in long rum among the diabetics.
\end{abstract}

Keywords: Diabetes mellitus, Glycated hemoglobin, serum ferritin, Insulin resistance.

Received: $26^{\text {th }}$ October, 2017

\section{Introduction}

A rise in the non communicable diseases is a serious threat globally. Among them diabetes mellitus ranks the top. By 2030, it is estimated that 79.4 million people will be in the grip of diabetes. It will be the seventh leading cause of death by 2030, as per the projection of WHO. An ICMR funded study in India pointed out that, south Indian population are more affected by diabetes than north Indian people. ${ }^{1}$ Cytokines and inflammatory factors play a pivotal role in producing Diabetes mellitus as said by Pick up JC. ${ }^{2}$ Metabolic disturbances associated with DM include hyperglycemia, insulin resistance, malnutrition, and other risk factors like hypertension, polycystic ovarian syndrome and dyslipidemia. Insulin resistance is associated with onset of type $2 \mathrm{DM}$ and various studies point its connection to iron overload in the body. Catalytic action of free iron is instrumental to insulin resistance in the beginning and later on to
Accepted: $26^{\text {th }}$ October, 2017

reduced insulin release which subsequently results in development of Type 2 DM. Long standing co morbidities due to diabetes are also moderated by iron mediated deterioration. ${ }^{3}$ Different studies across the globe and India have given inconsistent results regarding iron profile status in diabetics. Regulation of blood iron levels is mediated by Ferritin protein, Ferritin functions as a buffer in iron overload and iron overload. ${ }^{4}$ Ferritin is a complex globular protein that stores iron as soluble and non toxic component. Increasing concentration of iron and Ferritin in cells could cause resistance to insulin and dysfunction of $\beta$ cells of pancreas. Hyperinsulinemia due to resistance to insulin may be responsible for increasing serum Ferritin. Abnormalities in the Ferritin metabolism following glycation in chronic hyperglycemic state might be a cause of hyperferritenemia in $\mathrm{T} 2 \mathrm{DM}$ as mentioned in many studies universally. ${ }^{5}$ It is surmised that this increase may contribute to the pathogenesis of the disease and 
as well as development of complications like retinopathy, nephropathy etc. Serum concentrations of Ferritin are usually increased in poorly controlled type 1 and type 2 diabetic subjects, and Ferritin has been shown to predict $\mathrm{HbA1c}$ independently of glucose reflecting increased oxidative stress.

The present study was carried out to determine the relationship between serum Ferritin and type 2 diabetes mellitus and to establish correlation between serum Ferritin and fasting blood glucose as well as with HbA1c.

\section{Materials and Methods}

The present observational study was conducted at a tertiary care hospital in the department of biochemistry and research laboratory for a period of one year from October 2015 to September 2016. The study proposal was approved by the institutional ethical committee and all the ethical guidelines were followed as per the protocol. Written consent was obtained from all the cases and controls of the study after explaining the objectives of the study. The study population consisted of 50 cases of clinically diagnosed diabetics who were receiving oral hypoglycemic drugs and attending the department of endocrinology and general medicine and 50 controls of normal healthy subjects. The controls were age matched and all the cases and controls were males only in the study. All the cases and controls were evaluated with detailed clinical history, meticulous examination and laboratory investigations. The anthropometric measurements were noted i.e., age, weight, hip circumference and BMI was calculated. The data was collected and entered in a separate proforma sheet.

\section{Inclusion criteria}

Uncomplicated Type-2 diabetic male patients on oral hypoglycemic agents, with a history of diabetes less than 10 years and age greater than 25 years were included in the study.

\section{Exclusion criteria}

As Ferritin is an acute phase reactant, cases and controls with possible or suspected infection, inflammation or any other disease with a possible cause of elevation of serum Ferritin were excluded by estimation of CRP. Any case with raised CRP levels was excluded from the study. Patients with anemia, on iron supplementation therapy by any means, who underwent blood transfusion or donation, bleeding disorders and other diabetic complications (e.g. nephropathy, neuropathy etc) were excluded from the study.

\section{Sample collection}

$5 \mathrm{ml}$ of venous blood was collected after a period of 12 hours overnight fasting and FBS, $\mathrm{Hb} \%$, Serum Ferritin, Glycated hemoglobin (HbA1c) was estimated by standard protocols. $1 \mathrm{ml}$ of venous blood was collected only from cases after 2 hours post prandial and PPBS was measured.

1. Blood glucose was determined by Hexokinase G-6-PDH method and carried out on auto analyzer -Roche/Hitachi Cobas 6000 system. [Normal range : 70-110mg/dl] Post prandial blood glucose was also calculated by using the same auto analyzer. [Normal range 7-140 mg/dl]

2. Serum Ferritin was estimated by using DRG Ferritin ELISA kit which is a immune radiometric assay kit of the sand witch type using two monoclonal antibodies. Using the mean absorbance value, the corresponding concentration of Ferritin in $\mathrm{ng} / \mathrm{ml}$ was calculated from the standard curve. [Normal range: $20-250 \mathrm{ng} / \mathrm{ml}]$

3. HbA1c was measured by the Ion-exchange resin method, [Tulip group] [Normal value: $<6.5 \%$ ]

4. $\mathrm{Hb} \%$ was measured in whole blood by the cyanide free sodium lauryl sulphate method. [Normal range in males: 13-17 gm\%].

\section{Statistical Analysis}

Descriptive and inferential statistical analysis was carried out. Mean and standard deviation was carried out to assess the levels of various parameters in both groups. Student ' $t$ ' test was carried out for comparison of quantitative Co-relation between serum Ferritin and variables. Co-relation between serum Ferritin and HbA1c in patients and co-relation between serum Ferritin and FBS was evaluated by using Pearson correlation coefficient. P-valve $<0.05$ was considered statistically significant.

\section{Results}

In the present study conducted on diagnosed type 2 diabetic cases of males and controls, the highest age of diabetic male was 75 years and lowest age was 38 years, The most common age group was 41-50 years in both cases and controls. The mean age of diabetic cases was $48.56 \pm 6.75$ and control group was $46.28 \pm 9.75$ and there was no significant age difference in cases and control group. The BMI of all the diabetic cases were $>18.5 \mathrm{~kg} / \mathrm{m}^{2}$ and the mean BMI was $32.16 \pm 1.88 \mathrm{~kg} / \mathrm{m}^{2}$ and the BMI of control group was $28.22 \pm 2.68 \mathrm{~kg} / \mathrm{m}^{2}$ and less than the diabetic cases. Nearly $54 \%$ of diabetic 
cases had BMI between $25-30 \mathrm{~kg} / \mathrm{m}^{2}$ and was found statistically significant ( $\mathrm{p}$ value $<0.01$ ). The waist circumference and waist hip ratio of diabetics were higher than controls in the study indicating diabetics had central obesity. The waist to hip ratio is significantly higher ( $p$ value $>0.001)$ in diabetics $(1.12 \pm 0.12)$ than controls $(0.98 \pm 1.1)$ and diabetics are associated with central obesity. In the present study all the cases were diagnosed diabetics with FBS levels $>126 \mathrm{mg} / \mathrm{dl}$ and the mean value of FBS was 198 $\pm 46.1 \mathrm{mg} / \mathrm{dl}$ and in the controls was $98.6 \pm 2.1$ $\mathrm{mg} / \mathrm{dl}$ and was found to be statistically highly significant ( $\mathrm{p}$ value $<0.001$ ). The mean PPBS in the diabetic cases was higher $(286.12 \pm 2.65)$ than the control group $(118.12 \pm 2.1)$ and was found significant $(\mathrm{p}$ value $>0.01$ ). There was no much difference in the Hemoglobin $\%$ between the cases and controls in the study with a mean value of $15.12 \pm 1.1$ in cases and $14.98 \pm 1.4$ in controls and was not statistically significant. ( $p$ value $<0.01)$. The mean percentage of Glycated hemoglobin (HbA1c) in the controls was less $(5.01 \pm 0.45)$ than the percentage of HbA1c in cases $(9.48 \pm 2.65)$ and was found to be strongly significant in the study. The level of Serum Ferritin was higher in diabetic cases than controls in the study. Diabetics with a mean value of $359.11 \pm 87.18$ and in controls with a value of $182.12 \pm 26.14$ and showed a statistically significant association ( $\mathrm{p}$ value $<0.01$ ). In our study a rise in serum Ferritin levels were observed in diabetics even though the hemoglobin levels were in the normal physiological range. [Table-1]

In our study it was observed, serum Ferritin levels were higher $(214.14 \pm 5.85 \mathrm{ng} / \mathrm{ml})$ in diabetic cases with highest HbA1c levels of $>10.5 \%$. In contrary lower levels were observed $(89.45 \pm 2.14 \mathrm{ng} / \mathrm{ml})$ in cases with HbA1c levels between $6.0-7.5 \%$. [Table-2] Hence it was observed that levels of HbAlc had a strong positive correlation with levels of serum Ferritin in diabetic cases in our study and was statistically significant. The value of Pearson correlation coefficient overall was 0.98 and established a strong positive correlation between the two parameters in the study. [Table-3]

Table1: Comparision of means of anthropometric measurements, clinical and biochemical characteristics between test and control groups

\begin{tabular}{|l|c|c|c|}
\hline \multicolumn{1}{|c|}{ Parameters } & Control group & Diabetic group & p value \\
\hline Age $($ Years $)$ & $46.28 \pm 9.75$ & $48.56 \pm 6.75$ & $>0.05$ \\
\hline BMI $\left(\mathrm{kg} / \mathrm{m}^{2}\right)$ & $28.22 \pm 2.68$ & $32.16 \pm 1.88$ & $<0.01$ \\
\hline FBG $(\mathrm{mg} / \mathrm{dl})$ & $98.6 \pm 2.1$ & $198 \pm 46.1$ & $<0.001$ \\
\hline $\mathrm{PPBG}(\mathrm{mg} / \mathrm{dl})$ & $118.12 \pm 2.1$ & $286.12 \pm 2.65$ & $<0.01$ \\
\hline $\mathrm{Hb}(\%)$ & $14.98 \pm 1.4$ & $15.12 \pm 1.1$ & $<0.01$ \\
\hline $\mathrm{HbA} 1 \mathrm{c}(\%)$ & $5.01 \pm 0.45$ & $9.48 \pm 2.65$ & $<0.01$ \\
\hline Serum ferritin $(\mathrm{ng} / \mathrm{ml})$ & $182.12 \pm 26.14$ & $359.11 \pm 87.18$ & $<0.01$ \\
\hline
\end{tabular}

Table 2: Mean and Standard deviation of serum Ferritin in different levels of HbA1c among diabetic cases

\begin{tabular}{|l|c|c|c|}
\hline \multirow{2}{*}{$\begin{array}{c}\text { HbA1c (\%) } \\
\text { (Range) }\end{array}$} & \multirow{2}{*}{ No } & \multicolumn{2}{|c|}{ Mean \pm SD } \\
\cline { 3 - 4 } & & HbA1c (\%) & Ferritin (ng/dl) \\
\hline $6.00-7.50$ & 13 & $7.14 \pm 0.48$ & $89.45 \pm 2.14$ \\
\hline $7.51-9.00$ & 9 & $7.29 \pm 0.54$ & $144.12 \pm 14.12$ \\
\hline $9.01-10.5$ & 12 & $8.97 \pm 0.88$ & $181.15 \pm 21.01$ \\
\hline$>10.5$ & 16 & $11.21 \pm 1.14$ & $214.14 \pm 5.85$ \\
\hline
\end{tabular}

Table 3: Correlation between HbA1c and serum ferritin levels in Diabetic cases

\begin{tabular}{|l|c|c|}
\hline \multirow{2}{*}{ HbA1c Range } & \multicolumn{2}{|c|}{ Serum ferritin } \\
\cline { 2 - 3 } & r value & p Valve \\
\hline $6.00-7.50$ & 0.61 & $<0.05$ \\
\hline $7.51-9.00$ & 0.58 & $<0.05$ \\
\hline $9.01-10.5$ & 0.88 & $<0.01$ \\
\hline$>10.5$ & 0.74 & $<0.01$ \\
\hline Overall & $\mathbf{0 . 9 8}$ & $<0.01$ \\
\hline
\end{tabular}




\section{Discussion}

Chronic hyperglycemia is a major etiological factor which triggers both micro and macro vascular complications in diabetes mellitus. Persistent hyperglycemia in uncontrolled diabetics may cause low grade inflammation which result in increase of inflammatory markers which predispose to detrimental consequences in diabetes mellitus. The biochemical process of advanced glycation appears to be enhanced in diabetes as a result of hyperglycemia, oxidative stress and lipid peroxidation. In result of this, a different group of chemical moieties are generated which result in development and progression of vascular complications via activation of intracellular signaling pathways, generation of proinflammatory and prosclerotic cytokines. ${ }^{6,7} \mathrm{In}$ the present study, the most common age group of diabetic cases was 41-50 years followed by 5160 years which is similar to the findings of $\mathrm{Mc}$ Nair et al and Yoon. ${ }^{8,9}$

Serum ferritin is considered as a marker of iron status in the body and an inflammatory marker. Oxidative stress can increase ferritin synthesis to avoid further oxidative damages as ferritin can bind free redox-active iron. In the present study, the diabetic cases had increased waist hip ratio indicating an increase in BMI $\left(\mathrm{kg} / \mathrm{m}^{2}\right)$. In our study it was observed that $74 \%$ of diabetics were with central obesity with BMI greater than $25 \mathrm{~kg} / \mathrm{m}^{2}$.This findings were consistent with the findings of Wrede $\mathrm{Ce}$ et al. ${ }^{[10]}$ In the present study, the mean $\mathrm{HbA} 1 \mathrm{c} \%$ in diabetic cases was $9.48 \pm 2.65 \%$ and in the control group was $5.01 \pm 0.45 \%$ and the difference was statistically significant with $p$ value $<0.01$. Most of the studies earlier had reported that $\mathrm{HbAlc}$ is a good indicator of glycaemic control and highest values are found in cases with poorest control. ${ }^{[11]}$ An elevated level of $\mathrm{HbA1c}$ is associated with increased development and progression of micro vascular complications in patients with type 2 diabetes mellitus.

In the present study, serum ferritin levels are increased in diabetic cases than the controls. The mean serum ferritin concentration among diabetic cases was $359.11 \pm 87.18 \mathrm{ng} / \mathrm{ml}$ with $182.12 \pm 26.14 \mathrm{ng} / \mathrm{ml}$ in control group. Data of our study was almost similar to the findings of Ford et al, Sumesh $\mathrm{r}$ et al in their studies and also was statistically significant with $p$ value $<0.01 .^{12,13}$ All the linear regression coefficients between ferritin concentration, FBS, Hblac showed positive correlation and statistically significant. The present study indicates that serum ferritin was lowest in control group without diabetes and moderately raised in individuals with slightly elevated HBA1c levels and highest in cases with poorest glycaemic control with highly elevated $\mathrm{HbA} 1 \mathrm{c} \%$. Most of the studies globally have indicated that serum ferritin can be employed as a marker for not only glucose homeostasis but also insulin resistance in both type 2 diabetics and control subjects. In a study by Jiang et al, CRP was also adjusted along with serum ferritin in diabetic cases because ferritin reflects both the storage of iron as well as acute inflammation. ${ }^{14,15}$ Due to high levels of blood glucose in the cases of the study, there is an increased glucotoxicity which can contribute to oxidative stress and increased inflammation resulting consequently in higher serum ferritin levels. A significant positive correlation is seen between cases with high FBS, high PPBS, increased $\mathrm{HbA1c} \%$ and serum ferritin cases of the study. The prevalence of hyperferritenemia is $74.2 \%$ in the diabetic cases in the present study which is similar to the findings in the study of Meghna Borah et al. ${ }^{16}$

To conclude this study clearly explains that there is a significant increase in the serum ferritin in cases of diabetes mellitus compared to normal controls and hyperferritenemia may be one of the causes for decreased insulin production and development of insulin resistance in diabetic individuals. It was also observed that poorly controlled diabetic cases with elevated $\mathrm{HbA1c} \%$ had significant hyper ferritenemia. So it is suggestive that increased iron levels play a significant role in development of complications like microangiopathy, retinopathy in poorly controlled diabetics. Hence reliable and highly sensitive methods to detect free/catalytic iron that participates in tissue damage due to oxidative stress should be developed. Most of the studies reported that the levels of serum ferritin decreased after proper control of blood sugar levels which might be considered as one of the methods of diabetes indices control among diabetes subjects. ${ }^{[17]}$ Hence the study of iron and related parameters may be a useful offshoot of the conventional studies on diabetes and its complications. Hence the need for measures which can reduce the iron overload like phlebotomy and iron chelation therapy may be considered as a measure in prevention of complications in cases with poor glycemic control. Hence monitoring the prevalence of serum ferritin is beneficial in long rum among the diabetics. 


\section{References}

1. Anjana, R.M.; Ali, M.K.; Pradeepa, R.; Deepa, M.; Datta, M.; Unnikrishnan, R.; Rema, M.; Mohan, V. The need for obtaining accurate nationwide estimates of diabetes prevalence in India - Rationale for a national study on diabetes. Indian J. Med. Res. 2011,133,369-80.

2. Pickup JC, Crook MA. Is type II diabetes mellitus a disease of the innate immune system? Diabetologia.1998; 41:1241-8.

3. Kim, N.H.; Oh, J.H.; Choi, K.M.; Kim, Y.H.; Baik, S.H.;Choi, D.S.; Kim, S.J. Serum ferritin in healthy subjects and type 2 diabetic patients. Yonsei Med. J. 2000, 41,387-92.

4. Rachel C, Regi na F. Iron Use and Storage in the Body: Ferritin. [internet] Avaiable from: www.chemistry.wustl.edu/ edudev/ LabTutorials/ Ferritin/Ferritin.html.

5. Rajpathak S, Ma J, Manson J, Willett WC, Hu FB. Iron intake and the risk of type 2 diabetes in women: a prospective cohort study. Diabetes Care.2006;29:1370-6.

6. Srivatsan R, Das S, Gadde R, Manoj Kumar K, Taduri S, Rao N, et al Antioxidants and lipid peroxidation status in diabetic patients with and without complications. Arch Iranian Med. 2009; 12(2):121-27.

7. Goh SY, Cooper ME. The role of advanced glycation end products in progression and complications of diabetes. J Clin Endocrinol Metab. 2008;93:1143-52.

8. McNair P, Christiansen C, Christensen MS, Madsbad S, Faber OK. Development of bone mineral loss in insulin-treated diabetes: a 1 1/2 years follow-up study in sixty patients. Eur J Clin Invest.1981;11(1):55-9.

9. Yoon KH, Kim HS. A short message service by cellular phone in type2 diabetic patients for 12 months. Diabetes Res Clin Pract. 2008;79(2):25661.

10. Wrede CE, Buettner R, Bollheimer LC, et al. Association between serum ferritin and the insulin resistance syndrome in a representive population. Eur J Endocrinol 2006;154:333-40.

11. Fowler GC, Vasudevan DA. Type 2 diabetes mellitus: Managing hemoglobin A1cand beyond. Southern Med J. 2010;103(9):911-16.

12. Ford ES, Cogswell ME. Diabetes and serum ferritin concentration among U.S. adults. Diabetes Care.1999;22:1978-83.

13. Sumesh R, Rajan GV. Correlation between elevated serum ferritin and HbA1c in type 2 diabetes mellitus, Int J Res Med Sci. 2013 Feb;1(1):12-5.

14. Smotra S, Kudyar R.P. Relationship between Serum Ferritin and Type-2 Diabetes Mellitus. JK Science.2008;10(4):170-4.

15. Jiang R, Manson JE, Meigs JB, Ma J, Rifai N, Hu FB. Body iron stores in relation to risk of type 2 diabetes in apparently healthy women. JAMA.2004;291(6):711-7.

16. Borah M, Goswami RK. Evaluation of serum ferritin in in type II diabetes mellitus: a hospital based observational study from Dibrugarh, Assam, India. Int J Res Med Sci 2016;4:4916-21.

17. Kalantar-Zadeh K, Rodriguez RA, Humphreys $\mathrm{MH}$. Association between serum ferritin and measures of inflammation, nutrition and iron in hemodialysis patients. Nephrol. Dial. Transplant 2004;19(1):141-9. 